\title{
Editorial: Special Issue on "Social Innovation for Sustainable Infrastructure (SISI)"
}

\author{
Ramjee Prasad ${ }^{1} \cdot$ Marina Ruggieri $^{2}$ \\ Published online: 8 April 2019 \\ (c) Springer Science+Business Media, LLC, part of Springer Nature 2019
}

The 21st Edition of Strategic Workshop (SW'18) was held in, SCANDIC ISHAVSHOTEL TROMS $\varnothing$, Fredrik Langes gate 2, 9008 Troms $\varnothing$, Norvegia on May 14-16, 2018. The focus during the active 2-days discussion was Social Innovation for Sustainable Infrastructure (SISI) addressing concept, technology-oriented social innovation, business models for sustainability and humanity, applications, network security, and future strategy for nextgeneration internet.

The theme of the workshop was "Social Innovation for Sustainable Infrastructure (SISI)". In addition to the technical presentations on SISI, a primary goal of the workshop was to speed up innovation and the way to commercialization of the applicable technologies and a strong partnership among industry and academia. We concluded that modern practices and methodologies in the era of social science and technology are continuously unfolding. New innovative technologies and ICT are creating hindrance to make a regular or a distinct system of work to be followed. This uncertainty and constant transition require modification and our attention to addressing the issue. That abrupt change will abolish our modern system of work and way of thinking. The next major wave of information technology is all about combining the physical world with the Internet via pervasive deployment of mobile and embedded computing devices. The ability to view, explore and communicate with the physical world will intensify our lives in diverse ways. The result is a collection of evolving "wireless ecosystems" that are weaving wireless information into the fabric of human life and society.

To meet the objective as mentioned above, we organized a dedicated Panel and Sessions in the area of Social Innovation for Sustainable Infrastructure (SISI). During the workshop, we tried to investigate the digital metamorphosis, big data and smart concepts turmoil current industrial practices and business models. Several challenges for sustainability had

Chosen Topics from the Strategic Workshop, May 14-16, 2018, SCANDIC ISHAVSHOTEL TROMS $\varnothing$, Fredrik Langes gate 2, 9008 Troms $\varnothing$, Norvegia.

Ramjee Prasad

ramjee@btech.au.dk

Marina Ruggieri

ruggieri@uniroma2.it

1 Department of Business Development and Technology, Birk Centerpark 15, 8001, CGC, INNOVATORIUM, 7400 Herning, Denmark

2 Center for Teleinfrastructures (CTIF), University of Roma “Tor Vergata', Rome, Italy 
been addressed related to environment, policies and practices, ICT, operational assistance, connectivity and human resources. It had been stressed that the key challenge for sustainable infrastructure is to brew new coming innovation into a system, which is proactive, interdisciplinary, and building a prototype for sustainable infrastructure.

The Special Issue features selected fourteen papers that concentrate on a broad range of research challenges in Social Innovation for Sustainable Infrastructure (SISI) as well as addressing concerns about the application, services and security in coming wireless technologies and with this, technology-oriented innovative business models for Social Innovation for Sustainable Infrastructure and associated services.

The First Paper, "Effects of Correlated Multivariate FSO Channel on Outage Performance of Space-Air-Ground Integrated Network (SAGIN)", by Isiaka. A. Alimi, Antonio L. Teixeira, Paulo P. Monteiro.

The research paper has indicated how to share global information and resources efficiently over large network topology, the Space-Air-Ground Integrated Network (SAGIN) system that combines various networks for optimal utilization. In this paper, Authors examined the effect of correlated FSO channel on the SAGIN system outage performance.

To achieve this, they considered the exponential model for modelling the correlations between the apertures. Furthermore, to account for the spatial correlation for different diversity orders in the air-to-ground as well as air-to-air communications, they employed a multivariate Gamma-Gamma $(\Gamma \Gamma)$ distribution. The results of the analysis appropriately quantify the effects of the atmospheric turbulence-induced fading as well as correlation on the system outage performance.

The Second Paper, "Sustainable Framework for Smart Transportation System: A Case Study of Karachi", by Muhammad Aamir, Bai Ting Ting, Suhaib Masroor, Zain Anwar Ali.

The research paper represents a framework of smart transportation system striving to address the transportation problem in Karachi city. In this paper, Authors conducted a case study which highlights the current traffic situation of Karachi, and propose a framework to transform it into a smart transportation system. To have a smart transportation system, Authors have emphasized that it is necessary to have in-depth knowledge and information about the city dynamics and its traffic related issues. Finally, the Authors proposed a framework to develop a smart transportation system while keeping in mind the previous traffic problems.

The Third Paper, "Machine Learning for Wireless Communication Channel Modeling: An Overview", by Saud Aldossari, Kwang Cheng Chen.

Machine Learning for Wireless Communication Channel Modeling: An Overview.

The research paper is of the view that the Channel modeling is fundamental to design wireless communication systems. For highly mobile communications, channel estimation on top of the channel modeling enables high bandwidth physical layer transmission in state-of-the-art mobile communications. In this paper, the Authors applied the machine learning to assist channel modeling, and channel estimation has been introduced with evidence of literature survey.

The Fourth Paper, "Anti-Fog System for High-Speed Vehicles Using WI-FI", by Ghulam Fiza Mirza, A.W. Umrani, Abdul Razzaque Jawad, Nafeesa Bohra, B.S. Chowdhry, Fahim Aziz Umrani.

The paper discusses a novel offline system designed to drive the vehicles safely in a dense smoggy atmosphere where nothing is visible to the driver. The system utilizes off the shelf WI-FI modules operating in station mode for moving vehicles and access point (AP) mode for immobile vehicles to enable the driver to drive along a smoggy path in case if any 
vehicle is stopped on the motorway due to any reason (accident, car breakdown etc.) along with the use of NodeMcu (Microcontroller), Ultrasonic sensors and Accelerometer. The Authors have used the K-means clustering method using elbow technique to analyse the mobile phone's GPS coordinates and speed of the vehicle to do effective road monitoring. The proposed system would not only be helpful for the drivers to drive smoothly on the road in a foggy environment and avoid unforeseen catastrophes.

The Fifth Paper, "Why Cryptosystems Fail Revisited”, by Geir M. Køien.

In the paper, the Author has discussed "Why Cryptosystems Fail", Ross Anderson reflects on the question of why cryptosystems fail. There may be weak crypto-algorithms, short key lengths and flawed crypto-protocols, both by design and by the implementation. Nevertheless, these were not the main reason why cryptosystems failed. Anderson discovered that the problem had to do with misplaced trust and misconceptions of the threats the systems faced. The Author investigated the original paper and evaluated to what extent the situation is similar today.

The Sixth Paper, "Micro and Macro Network Slicing: an experimental assessment of the impact of increasing numbers of slices", by Flavio Meneses, Rui Silva, Daniel Corujo, Rui L. Aguiar.

In this paper, the Authors investigated the challenges associated to a vision where the network slicing concept is applied with a much greater level of granularity, ultimately allowing it to become a core mechanism of the network's operation, with large numbers of co-existing slices. In that respect, this paper proposes an architecture framework for instantiation of network slices among network providers, which in turn can instantiate sub-slices tailored to use cases and vertical tenants. The evaluation of this concept is done following a two-pronged approach: firstly, different slice dimensions (i.e., from micro to macro) are proposed and discussed, pointing out the benefits and challenges of each proposed slice; secondly, we deployed a mobile network provider (MNO), using OpenAirInterface and FlexRAN frameworks, and experimentally evaluated its slicing mechanisms. The objective is to provide insight into the challenges and impact associated with the deployment of an increasing number of slices, using the same available infra-structural resources.

The Seventh Paper, "Resource Reuse for D2D Communication in LTE Networks by Efficient Grouping”, by Fang-Chang Kuo, Hwang-Cheng Wang, Kuo-Chang Ting, Jia-Hao $\mathrm{Xu}$, Chih-Cheng Tseng.

The Authors explained that the Device to Device (D2D) communication is a key technology of 5G mobile communications. To efficiently use the limited resource blocks (RB), the Authors suggested that D2D pairs can mutually tolerate the induced interference are grouped into a clique and reuse the assigned RBs. Then, the system capacity can be improved as a result. Previous methods evaluated SINR by employing device locations and free space path loss formulae. Between the two devices of a D2D pair, however, there may be obstacles which lead to severe signal attenuation. To deal with this problem, we propose a practical approach which evaluates the SINR by employing the Reference Signals Received Power (RSRP) reported by the devices during the proximity discovery phase. We also use some methods to improve the performance of the system.

The Eighth Paper, "Enabling generic wireless coexistence through technology-agnostic dynamic spectrum access", by Irfan Jabandzic, Spilios Giannoulis, Ingrid Moerman.

Wireless research teams targeting heterogeneous wireless communication coexistence are developing techniques for enabling one-to-one coexistence between various wireless technologies. The Authors believe that a one-to-one approach is inefficient and cannot lead to a generic coexistence paradigm, applicable to every existing or new wireless communication technology that will arise in the future. In this paper, the Authors 
presented a new generic medium sharing model that solves the vast coexistence problems observed today quietly and efficiently.

The Ninth Paper, "Edge-node-aware Adaptive Data Processing Framework for Smart Grid", by Isma Farah Siddiqui, Nawab Muhammad Faseeh Qureshi, Muhammad Aslam Uqaili, Bhawani Shankar Chowdhry.

In this paper, the Authors presented an Edge-node-aware framework that empowers nodes to process IoT and counterpart data intelligently. Newly, it is observed that the grid infrastructure is rapidly transforming network topology towards green and eco-friendly computing. For this, the edge-computing environment is being adopted to inter-connect distributed IoT nodes in peer-to-peer sequential order. The Authors recommended by this an approach, the grid ideally cope with green computing issues than traditional infrastructure, nevertheless, it faces specific data processing problems such as differentiating IoT and non-IoT data segments, storing data chunk with end of file (EOF) assurance, management of replica data segments and systematic analytics of edge node datasets.

The Tenth Paper, "A Telemedicine Platform for Disaster Management and Emergency Care”, by Sadia Anwar, Ramjee Prasad, Bhawani Shankar Chowdhary, M. R. Anjum.

In this paper, the Authors proposed a model where telemedicine technology could be helpful especially in the areas where the shortage of medical specialist or doctors has happened due to a disaster situation. The Authors suggested that the Distance from approaching a victim and waiting time can be lessened by a fight-or-flight strategy which is active and rebound since it demands coordination and communication between various sectors for compliance and rebuilding with an intelligent strategy. Telemedicine applications in disaster situation require efficient, reliable communication technology such as GPRS, LTE etc. However, transmission losses or delay occur during transmission. The Authors proposed that by using Friis transmission equation, condition transmission falls can be minimized for efficient communication.

The Eleventh Paper, "Functional Split Perspectives: A Disruptive Approach to RAN Performance Improvement", by Isiaka. A. Alimi, Paulo P. Monteiro.

In this paper, the Authors offers a comprehensive overview of the traditional C-RAN MFH and considers some viable RAN FunStiOn techniques that can help in relaxing the stringent requirements. The related trade-offs of the considered RAN FunStiOns are also presented. Also, the Authors offered and discussed many relevant, cost-effective architectural variants that can relatively simplify the DU designs with low-footprints. The Authors have presented architectural variants that can be employed to support different use cases of the emerging $5 \mathrm{G}$ networks depending on the deployment scenarios.

The Twelfth Paper, "Blockchain paradigm and Internet of Things", by Valentin Rakovic, Jovan Karamachoski, Vladimir Atanasovski, Liljana Gavrilovska.

In this paper, the Authors elaborates the $\mathrm{BC}$-IoT related issues and provides a comprehensive survey of the current literature and relevant initiated deployments. The paper also identifies the key research and development challenges and discusses the possible aspects for future research. However, the core BC technology is computationally expensive and commonly involves high bandwidth overhead and delays that are not suitable for IoT related scenarios. To foster the synergy between the BC and IoT, recent research advancements have specifically focused on developing novel BC approaches that are tailored to the requirements and needs of the specific IoT use cases.

The Thirteenth Paper, "Vehicular channels: characteristics, models and implications on communication systems design", by Jose A. Cortes, Mari Carmen, Aguayo-Torres, Francisco J. Canete, Gerardo Gomez, Eduardo Martos-Naya, J. Tomas Entrambasaguas. 
In this paper, the Authors analyzes the relation between the characteristics of vehicular communication channels and the parameters of the referred systems, with particular emphasis on the physical and medium access control (MAC) layers. The Authors elaborated about the primary factors that influence V2V and V2I channels, and their main characteristics are first described. Illustrative results for a highway scenario, as well as a summary of the channel parameters reported in the literature, are given. The employed modeling approaches are then reviewed, and a representative model of the two main strategies is provided.

The Fourteenth Paper, "Security for Smart Grid in 5G and Beyond Networks", by Sarmistha De Dutta, Ramjee Prasad.

In this paper, the Authors have explained that The Smart Grid is the next-generation electrical power system that combines OT (Operations Technology) and IT (Information Technology) for the efficient generation, delivery, and consumption of electrical energy. The Authors aimed to provide a brief overview M2M communication and its history, its application in the Smart Grid, security issues affecting M2M data on the Smart Grid and some available solutions to detect and prevent cyber threats.

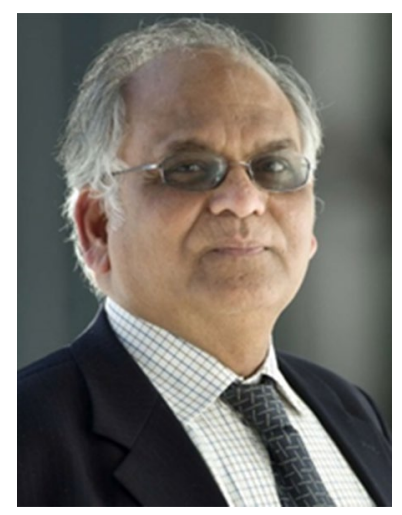

Dr. Ramjee Prasad is a Professor of Future Technologies for Business Ecosystem Innovation (FT4BI) in the Department of Business Development and Technology, Aarhus University, Denmark. He is the Founder President of the CTIF Global Capsule (CGC). He is also the Founder Chairman of the Global ICT Standardization Forum for India, established in 2009. GISFI has the purpose of increasing the collaboration between European, Indian, Japanese, North-American and other worldwide standardization activities in the area of Information and Communication Technology (ICT) and related application areas. He has been honoured by the University of Rome "Tor Vergata", Italy as a Distinguished Professor of the Department of Clinical Sciences and Translational Medicine on March 15, 2016. He is Honorary Professor of University of Cape Town, South Africa, and University of KwaZulu-Natal, South Africa. He received Ridderkorset af Dannebrogordenen (Knight of the Dannenberg) in 2010 from the Danish Queen for the internationalization of top-class telecommunication research and education. He has received several international awards such as: IEEE Communications Society Wireless Communications Technical Committee Recognition Award in 2003 for making contribution in the field of "Personal, Wireless and Mobile Systems and Networks", Telenor's Research Award in 2005 for impressive merits, both academic and organizational within the field of wireless and personal communication, 2014 IEEE AESS Outstanding Organizational Leadership Award for: "Organizational Leadership in developing and globalizing the CTIF (Center for TeleInFrastruktur) Research Network", and so on. He has been Project Coordinator of several EC projects namely, MAGNET, MAGNET Beyond, eWALL and so on. He has published more than 30 books, 1000 plus journal and conference publications, more than 15 patents, over 100 Ph.D. Graduates and a more significant number of Masters (over 250). Several of his students are today worldwide telecommunication leaders themselves. Under his leadership, magnitudes of close collaborations are being established among premier universities across the globe. The collaborations are regulated by guidelines of the Memorandum of Understanding (MoU) between the collaborating universities. 


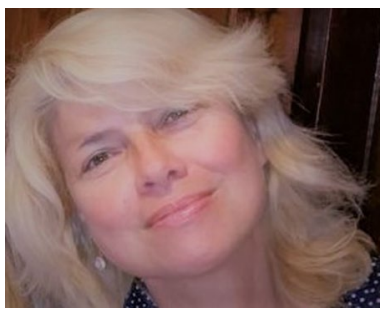

Marina Ruggieri is Full Professor of Telecommunications Engineering at the University of Roma "Tor Vergata", therein: member of the Board of Directors; Chair of the President's Advisory Committee for the relationship with the Italian Space Agency (ASI); co-founder and Steering Board Steering Board Chair of CTIF-an Interdisciplinary Research Center on Information and Communications Technology and its verticals, that belongs to the a broad research network, with nodes in USA, Europe and Asia. She has been: Vice President with responsibility of the ASI Technical and Scientific Council and member of the Research Policy Experts Advisory Committee of the University and Research Ministry. She is arbitrator of the Italian Industries Federation for Aerospace, Defense and Security (AIAD). She has been member of the Technical-Scientific Committee of the Center for Aeronautical Military Studies and Vice President of the Roma Chapter of AFCEA. She is Principal Investigator of the 40/50 GHz TPD\#5 Communications Experiment on board Alphasat (launched on July 2013). She is IEEE Aerospace and Electronic Systems Society (AESS) Officer and member of the Board of Governors (2019-2021). She has been IEEE 2018 Chair, TAB Strategic Planning Committee; 2017 Vice President, Technical Activities; 2014-2015 Director of IEEE Division IX; 2010-2011 AESS President. She has been member of many IEEE Committees, including Public Visibility, Fellow, Governance, Nominations and Appointments, Women in Engineering, Awards Board, and of the IEEE Board of Directors. She is recipient of 1990 Piero Fanti International Prize; 2009 Pisa Donna Award for women in engineering; 2013 Excellent Women in Roma Award; 2011 AESS Service Award. She has been inducted as a Professional into the IEEE Honor Society Eta Kappa Nu (HKN). She is IEEE Fellow "for contributions to millimeter-wave satellite communications" She is author/co-author of 335 papers, 1 patent and 12 books. 